\title{
Selection rules in rare earth MCD spectra. An experimental confirmation
}

\author{
Görller-Walrand, C.; Behets, M.; Porcher, P.; Laursen, Ib
}

Published in:

Journal of Chemical Physics

Link to article, DOI:

10.1063/1.449046

Publication date:

1985

Document Version

Publisher's PDF, also known as Version of record

Link back to DTU Orbit

Citation (APA):

Görller-Walrand, C., Behets, M., Porcher, P., \& Laursen, I. (1985). Selection rules in rare earth MCD spectra. An experimental confirmation. Journal of Chemical Physics, 83(9), 4329-4337. https://doi.org/10.1063/1.449046

\section{General rights}

Copyright and moral rights for the publications made accessible in the public portal are retained by the authors and/or other copyright owners and it is a condition of accessing publications that users recognise and abide by the legal requirements associated with these rights.

- Users may download and print one copy of any publication from the public portal for the purpose of private study or research.

- You may not further distribute the material or use it for any profit-making activity or commercial gain

- You may freely distribute the URL identifying the publication in the public portal

If you believe that this document breaches copyright please contact us providing details, and we will remove access to the work immediately and investigate your claim. 


\section{Selection rules in rare earth MCD spectra. An experimental confirmation}

C. Görller-Walrand and M. Behets

Laboratory of Inorganic Chemistry, Department of Chemistry, University of Leuven, Celestijnenlaan 200F, 3030 Heverlee, Belgium

P. Porcher

Laboratoire des Eléments de Transition dans les Solides, E. R. 210 du CNRS, 1 Place A. Briand, 92195 Meudon Principal Cedex, France

I. Laursen

Department of Electrophysics, The Technical University of Denmark, 2800 Lyngby, Denmark

(Received 27 December 1984; accepted 23 May 1985)

The MCD spectra of the ${ }^{5} D_{1} \leftarrow{ }^{7} F_{0}$ and ${ }^{5} D_{2} \leftarrow{ }^{7} F_{0}$ transitions are analyzed for two complexes of the $\mathrm{Eu}^{3+}$ ion with the $\mathrm{F}^{-}$ligand: the $\mathrm{LiYF}_{4} / \mathrm{Eu}^{3+}\left(D_{2 d}\right.$ symmetry $)$ and $\mathrm{KY}_{3} \mathrm{~F}_{10} / \mathrm{Eu}^{3+}\left(C_{4 v}\right.$ symmetry). The sign inversion of the $\mathscr{A}$ terms, predicted theoretically in a previous paper, is confirmed experimentally. It is shown how MCD is able to distinguish unambiguously between the $C_{4 v}$ and the $D_{2 d}$ coordination symmetries.

\section{INTRODUCTION}

The aim of this paper is to give an experimental confirmation of the selection rules, derived theoretically in previous papers, ${ }^{1,18}$ for the MCD spectra of rare earths, in the light of two mechanisms which account mainly for the spectral intensities of rare earth absorption bands, namely, the magnetic dipole and the induced electric dipole mechanisms.

The MCD experiments were performed on the $\mathrm{Eu}^{3+}$ ion complexes with a common ligand $\left(\mathrm{F}^{-}\right)$but in two different coordination symmetries $\left(D_{2 d}\right.$ and $\left.C_{4 v}\right)$.

In order to confirm the sign inversion predicted previously on a theoretical basis ${ }^{1}$ we analyzed for both symmetries the properties of the Faraday $\mathscr{A}$ terms corresponding to the ${ }^{5} D_{1} \leftarrow \leftarrow^{7} F_{0}$ and ${ }^{5} D_{2} \leftarrow^{7} F_{0}$ transitions where, respectively, the magnetic dipole and induced electric dipole character are predominant. ${ }^{19}$

In the first section of the paper we recall the theoretical background of the subject making exclusive use of the symbols, definitions, and sign conventions of two papers, one on MCD by Stephens in $1976,{ }^{4}$ (and subsequently recommended in the recent monograph by Piepho and Schatz ${ }^{5}$ ), and the other one by $\mathrm{Judd}^{2}$ on the absorption intensities of rare earth ions.

The central objective of this paper is to look at the MCD spectra of rare earths in the framework of the Judd-Ofelt theory ${ }^{2,6}$ and to show how magnetic circular dichroism through the sign properties of its $\mathscr{A}, \mathscr{B}$, and $\mathscr{C}$ parameters is much more sensitive to site symmetry effects than absorption or emisson signals.

\section{THEORY}

The MCD circular dichroic signal $\Delta A$ is expressed by (see formula 44 in Ref. 4):

$$
\begin{aligned}
\Delta A= & A_{-}-A_{+} \\
= & \gamma \mathscr{E}\left\{\mathscr{A}_{1}\left(-\frac{\partial f(\mathscr{E})}{\partial \mathscr{E}}\right)\right. \\
& \left.+\left(\mathscr{B}_{0}+\frac{\mathscr{C}_{0}}{k T}\right) f(\mathscr{E})\right\} \beta H C z,
\end{aligned}
$$

where

$$
\begin{aligned}
\mathscr{A}_{1}= & \frac{1}{d_{A}} \sum_{\alpha, \lambda}\left[\left|\left\langle A_{\alpha}\left|0_{-}\right| J_{\lambda}\right\rangle^{0}\right|^{2}-\left|\left\langle A_{\alpha}\left|0_{+}\right| J_{\lambda}\right\rangle^{0}\right|^{2}\right] \\
& \times\left[\left\langle J_{\lambda}\left|L_{z}+2 S_{z}\right| J_{\lambda}\right\rangle^{0}\right. \\
& \left.-\left\langle A_{\alpha}\left|L_{z}+2 S_{z}\right| A_{\alpha}\right\rangle^{0}\right] \\
\mathscr{B}_{0}= & \frac{2}{d_{A}} \sum_{\alpha, \lambda} \operatorname{Re}\left\{\sum _ { K _ { \kappa } \neq J } \left[\left\langle A_{\alpha}\left|0_{-}\right| J_{\lambda}\right\rangle^{0}\left\langle K_{\kappa}\left|0_{+}\right| A_{\alpha}\right\rangle^{0}\right.\right. \\
& \left.-\left\langle A_{\alpha}\left|0_{+}\right| J_{\lambda}\right\rangle^{0}\left\langle K_{\kappa}\left|0_{-}\right| A_{\alpha}\right\rangle^{0}\right] \\
& \times \frac{\left\langle J_{\lambda}\left|L_{z}+2 S_{z}\right| K_{\kappa}\right\rangle^{0}}{W_{K}^{0}-W_{J}^{0}} \\
& +\sum_{K_{\alpha} \neq A}\left[\left\langle A_{\alpha}\left|0_{-}\right| J_{\lambda}\right\rangle^{0}\left\langle J_{\lambda}\left|0_{+}\right| K_{\kappa}\right\rangle^{0}\right. \\
& \left.-\left\langle A_{\alpha}\left|0_{+}\right| J_{\lambda}\right\rangle^{0}\left\langle J_{\lambda}\left|0_{-}\right| K_{\kappa}\right\rangle^{0}\right] \\
& \left.\times \frac{\left\langle K_{\kappa}\left|L_{z}+2 S_{z}\right| A_{\alpha}\right\rangle^{0}}{W_{K}^{0}-W_{A}^{0}}\right\}
\end{aligned}
$$

with $A_{-}$and $A_{+}$: absorbance for rep and lcp (right circularly polarized light and left circularly polarized light).

$$
\mathscr{E} \text { : energy }
$$$$
\gamma=x \frac{\mathscr{N} \pi^{2} \log _{10} \mathrm{e}}{250 \hbar c} \text { with }
$$

$\mathscr{N}$ : Avogadro's number,

$x$ : effective field correction,

$x=\alpha^{2} / n$ for electric dipole transitions,

$x=n$ for magnetic dipole transitions,

$\alpha$ : electric permeability,

$n$ : refractive index.

$f(\mathscr{C})$ : shape function so that $\int f(\mathscr{C}) d \mathscr{E}=1$, $\mathscr{A}_{1}, \mathscr{B}_{1}, \mathscr{C}_{0}$ : Faraday parameters,

$k$ : Boltzmann constant,

$T$ : temperature,

$\beta$ : Bohr magneton, 
or

$H$ : magnetic field,

$C$ : concentration,

$z$ : optical pathway,

$0_{ \pm}=\mp(1 / \sqrt{ } 2)\left(\mathrm{m}_{\mathrm{x}} \pm \mathrm{im}_{\mathrm{y}}\right)$ : electric dipole operator for lcp and rcp

$0_{ \pm}=\mp(1 / \sqrt{ } 2)\left(\mu_{x} \pm i \mu_{y}\right):$ magnetic dipole operator for lcp and rcp,

$d_{A}$ : degeneracy of the ground state,

$A_{\alpha}$ : zero field (absence of magnetic field) wave

functions for the ground state.

If $A$ is degenerate $\alpha=1$ to $d_{A}$.

$J_{\lambda}$ : zero field wave functions for the excited state.

If $J$ is degenerate $\lambda=1$ to $d_{J}$.

$K_{\kappa}$ : zero field functions for the excited state,

$W_{A}^{0}, W_{J}^{0}, W_{K}^{0}$ : electronic state energies,

$\left(L_{z}+2 S_{z}\right) B H:-\mu_{z} H:$ Zeeman operator,

$\gamma^{0}:$ the superscript zero's designate zero field quantities,

$\mathscr{A}_{1}$ : the subscript 1 designates a first order moment quantity,

$\mathscr{B}_{0}$ : the subscript 0 designates a zero order moment quantity.

Note: In order to account for MCD it is convenient to choose unperturbed wave functions which are diagonal in the Zeeman operator.

\section{A. Sign convention}

Following Piepho and Schatz ${ }^{5}$ the CD sign is positive when absorption for left circularly polarized light is greater than for right circularly polarized light. As a consequence of having a positive $\mathscr{A}_{1}$ term, the absorption of left circularly polarized light must take place at higher energy than absorption of right circularly polarized light (see Fig. 1). A positive $\mathscr{B}$ term corresponds to absorption of left circularly polarized light.

\section{B. The expression for the $\mathscr{A}_{1}$ term in the Judd's notation}

The expression for the $\mathscr{A}_{1}$ term becomes ${ }^{2}$

$$
\begin{aligned}
\mathscr{A}_{1}= & \frac{1}{d_{A}} \sum_{\alpha, \alpha^{\prime}}\left[\left|\left\langle A_{\alpha}\left|0_{-}\right| A_{\alpha^{\prime}}^{\prime}\right\rangle^{0}\right|^{2}-\left|\left\langle A_{\alpha}\left|0_{+}\right| A_{\alpha^{\prime}}^{\prime}\right\rangle^{0}\right|^{2}\right] \\
& \left.\times\left[\left\langle A_{\alpha^{\prime}}^{\prime}\left|L_{z}+2 S_{z}\right| A_{\alpha^{\prime}}^{\prime}\right\rangle^{0}\right]-\left\langle A_{\alpha}\left|L_{z}+2 S_{z}\right| A_{\alpha}\right\rangle^{0}\right],
\end{aligned}
$$

where $A$ and $A^{\prime}$ are the ground and excited crystal field levels which correspond to

$$
\left\langle A_{\alpha}\right| \equiv \sum_{M}\left\langle 1^{N} \psi J M\right| a_{M}
$$

and

$$
\left|A_{\alpha^{\prime}}^{\prime}\right\rangle \equiv \sum_{M^{\prime}} a_{M^{\prime}}^{\prime}\left|1 \psi^{\prime} J^{\prime} M^{\prime}\right\rangle
$$

If in addition to $J$ and $J^{\prime}, M$ and $M^{\prime}$ are still good quantum numbers for the crystal field levels an obvious simplification results in the expression of the $\mathscr{A}_{1}$ term since the summation of the $M$ or $\mathbf{M}^{\prime}$ terms reduces to one term $\left(a_{M}=1\right.$ and $a_{M}^{\prime}$,
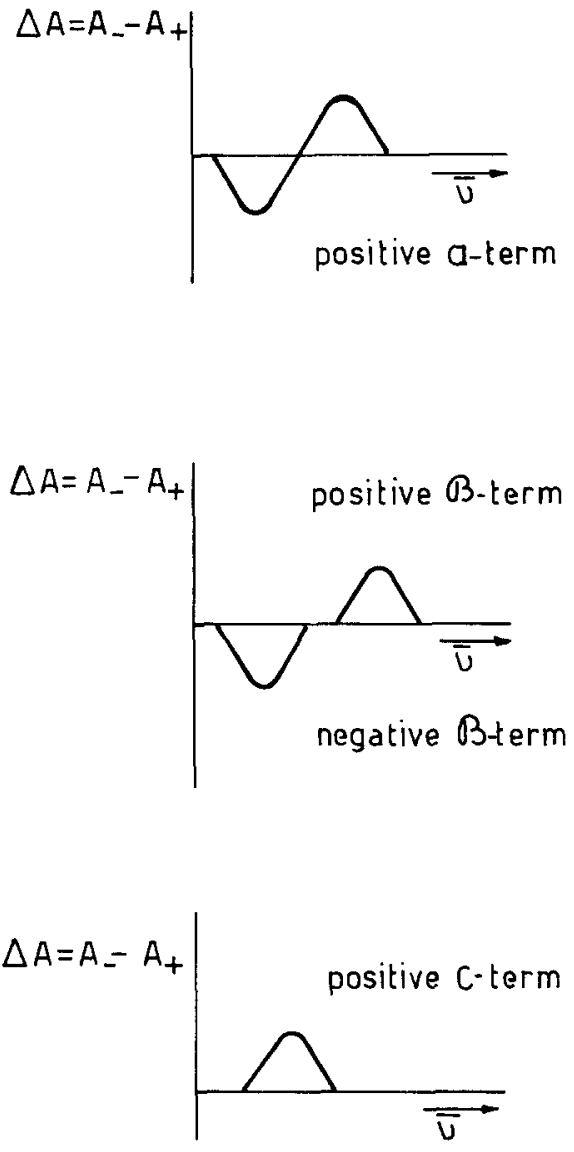

FIG. 1. Sign convention.

$=1$ 1). Also, the summation over the $\alpha$ or $\alpha^{\prime}$ terms reduces maximally to two terms corresponding to the components with quantum numbers $|\boldsymbol{M}|,-|\boldsymbol{M}|$ and $\left|M^{\prime}\right|,-\left|M^{\prime}\right|$; the cubic and icosahedral symmetries are not considered in this treatment (for details see Ref. 18).

If, moreover, the ground level is nondegenerate, and necessarily labeled by $M=0$ (otherwise $M$ will no longer be a good quantum number for the crystal field level), the expression of the $\mathscr{A}_{1}$ term becomes, in the simplified notation $\langle A| \equiv\langle J 0|$ and $\left|A^{\prime}\right\rangle \equiv\left|J^{\prime} M^{\prime}\right\rangle$,

$$
\begin{aligned}
& \mathscr{A}_{1} \sum_{\left.+\mid M^{\prime}\right)_{0}-\left|M^{\prime}\right|}\left[\left|\left\langle J 0\left|0_{-}\right| J^{\prime} M^{\prime}\right\rangle\right|^{2}-\left|\left\langle J 0\left|0_{+}\right| J^{\prime} M^{\prime}\right\rangle\right|^{2}\right] \\
& \quad \times\left[\left\langle J^{\prime} M^{\prime}\left|L_{z}+2 S_{z}\right| J^{\prime} M^{\prime}\right\rangle\right] .
\end{aligned}
$$$$
\text { As }
$$

$\sum_{+\left|M^{\prime}\right|,-\left|M^{\prime}\right|}\left|\left\langle J 0\left|0_{-}\right| J^{\prime} M^{\prime}\right\rangle\right|^{2}$

$$
=\sum_{+\left|M^{\prime}, \cdot-\right| M^{\prime} \mid}\left|\left\langle J 0\left|0_{+}\right| J^{\prime} M^{\prime}\right\rangle\right|^{2}
$$

and

$$
\begin{aligned}
\left\langle J^{\prime},\right. & \left.+\left|M^{\prime}\right|\left|L_{z}+2 S_{z}\right| J^{\prime},+\left|M^{\prime}\right|\right\rangle \\
& =-\left\langle J^{\prime},-\left|M^{\prime}\right|\left|L_{z}+2 S_{z}\right| J^{\prime},-\left|M^{\prime}\right|\right\rangle .
\end{aligned}
$$

In Russell-Saunders coupling:

$$
\left\langle J^{\prime}, \pm\left|M^{\prime}\right|\left|L_{z} \mathrm{~S}_{z}+2 \mathrm{~S}_{z}\right| J^{\prime}, \pm\left|M^{\prime}\right|\right\rangle= \pm\left|M^{\prime}\right| g
$$

with 
$g=1+\frac{J(J+1)-L(L+1)+S(S+1)}{2 J(J+1)}:$ (the Landé factor).

One subsequently obtains for $\mathscr{A}_{1}$ the expression $\mathscr{A}_{1}=2\left|\left\langle J 0\left|0_{-}\right| J^{\prime} M^{\prime}\right\rangle\right|^{2}\left[\left\langle J^{\prime} M^{\prime}\left|\mathrm{L}_{z}+2 \mathrm{~S}_{z}\right| J^{\prime} M^{\prime}\right\rangle\right]$.

If $g$ is positive the sign of $\mathscr{A}_{1}$ is determined by the sign of $M^{\prime}$ used to label the Zeeman component to which left circularly polarized light is absorbed. If $g$ is negative the opposite statement holds true.

We will now consider this rule in the light of the two mechanisms that account predominantly for the intensity in the spectra of rare earth complexes, i.e., the magnetic dipole and the induced electric dipole mechanisms.

(a) Magnetic dipole transition $\left[0_{-}=\mu_{-1}\right.$ $\left.=-(|e| / 2 m c)(L+2 S)_{-1}^{(1)}\right]$.

For a magnetic dipole transition the intensity matrix element is related to

$$
\begin{aligned}
& \left|\left\langle J 0\left|(L+2 S)_{q}^{(1)}\right| J^{\prime} M^{\prime}\right\rangle\right|^{2} \\
& =\left|(-1)^{J}\left(\begin{array}{lll}
J & 1 & J^{\prime} \\
0 & q & M^{\prime}
\end{array}\right)\left\langle J|| L+2 S|| J^{\prime}\right\rangle\right|^{2},
\end{aligned}
$$

where $q=-1$ and +1 for left and right circularly polarized light. The selection rule for a magnetic dipole transition can be derived from the $3 j$ symbol:

$$
M^{\prime}=-q=-(-1) \text {. }
$$

This means that absorption of left circularly polarized light occurs to $M^{\prime}=+1$ giving always a positive $\mathscr{A}_{1}$ term ifg is positive.

(b) Induced electric dipole transition $\left(0_{-}=m_{-1}\right.$ $\left.=-|e| D_{q}^{(1)}\right)$.

For an induced electric dipole transition, from Eq. (13) in Ref. 2, the intensity matrix element is related to

$$
\begin{aligned}
\left|\left\langle J 0\left|D_{q}^{(1)}\right| J^{\prime} M^{\prime}\right\rangle\right|^{2} & \\
= & \mid \sum_{p, r, \text { even }}(2 \lambda+1)(-1)^{p+q} \\
& \times A_{t p}\left(\begin{array}{ccc}
1 & \lambda & t \\
q & -p-q & p
\end{array}\right) \Xi(t, \lambda) \\
& \times\left.\left\langle J 0\left|U_{p+q}^{(\lambda)}\right| J^{\prime} M^{\prime}\right\rangle\right|^{2} .
\end{aligned}
$$

This expression may be further reduced to $\left|\left\langle J 0\left|D_{q}^{(1)}\right| J^{\prime} M^{\prime}\right\rangle\right|^{2}$

$$
\begin{aligned}
= & \mid \sum_{p, t, \text { even }}(2 \lambda+1)(-1)^{p+q} A_{t p} \\
& \times\left(\begin{array}{ccc}
1 & \lambda & t \\
q & -p-q & p
\end{array}\right) \Xi(t, \lambda) \\
& \times\left.(-1)^{J}\left(\begin{array}{ccc}
J & \lambda & J^{\prime} \\
0 & p+q & M^{\prime}
\end{array}\right)\left\langle J|| U^{(\lambda)}|| J^{\prime}\right\rangle\right|^{2} .
\end{aligned}
$$

Besides a series of selection rules on $J, \lambda, t \ldots$ given in Refs. 2 , 18 , and 19 , there is one that follows from the second $3 j$ symbol and which is relevant for MCD, namely,

$$
M^{\prime}=-(p+q) \text {. }
$$

From Eq. (7) the sign of the $\mathscr{A}$ term is thus determined by the sign of the $M^{\prime}$ value that satisfies the $3 j$ symbol for $q=-1$.
The sign of this $M^{\prime}$ value is clearly related to the symmetry of the coordination sphere around the rare earth ion through the $p$ value which appears in the odd crystal field terms

$$
V=\sum_{t, p} A_{t p} D_{p}^{(t)} .
$$

\section{EXPERIMENTAL}

\section{A. Choice of the single crystals and synthesis}

To confirm the previously deduced selection rules and demonstrate their influence on the sign of the $\mathscr{A}_{1}$ term, it was necessary to find a case where a given lanthanide ion could be complexed with the same ligand but in different coordination symmetries. The phase diagram of the system $R F-Y_{3}$ doped with $\mathrm{Eu}^{3+}$ (R: alkaline ion $\mathrm{K}$ or $\mathrm{Li}$ ) suggests the possibility of synthesizing two types of complexes: $\mathrm{RY}_{3} \mathrm{~F}_{10} / \mathrm{Eu}^{3+}$ with $C_{4 v}$ coordination symmetry around the rare earth, and the $\mathrm{RYF}_{4} / \mathrm{Eu}^{3+}$ with $D_{2 d}$ symmetry. For practical synthesis reasons $R Y_{3} F_{10}$ was prepared with the $K$ cation $\left(\mathrm{KY}_{3} \mathrm{~F}_{10} / \mathrm{Eu}^{3+}\right)^{7}$ while the $\mathrm{RYF}_{4}$ was obtained with the $\mathrm{Li}$ cation $\left(\mathrm{LiYF}_{4} / \mathrm{Eu}^{3+}\right) .8$

\section{B. Crystal structure and relation to site symmetry}

The $\mathrm{LiYF}_{4} / \mathrm{Eu}^{3+}$ system crystallizes in the tetragonal scheelite structure (space group $C_{4 h}^{6}$ ). The polyhedron formed by the nearest neighbors of the rare earth ion, eight fluorides $\left(\mathrm{F}^{-}\right)$, is nearly a dodecahedron $\left(D_{2 d}\right)$ (see Fig. 2). There is a slight lowering in symmetry to $S_{4}$ which implies a small distortion of the $\varphi$ coordinate.

The $\mathrm{KY}_{3} \mathrm{~F}_{10} / \mathrm{Eu}^{3+}$ complex crystallizes in a cubic system $(F m 3 m) \cdot{ }^{10}$ In this structure which is derived from the fluorite structure each rare earth ion is surrounded by eight $\mathrm{F}^{-}$ions forming a slightly distorted square-based antiprism $\left(C_{4 v}\right)$.

\section{Orientation of the crystals}

The $\mathrm{LiYF}_{4} / \mathrm{Eu}^{3+}$ was cut and polished as $\mathrm{a} \pm 1 \mathrm{~mm}$ slab perpendicular to the optical tetragonal $c$-crystal axis which corresponds to the $S_{4}$ axis in the symmetry and oriented with this $S_{4}$ axis $\|$ to the magnetic field. The application of the magnetic field $\left(H_{2}\right)$ on the $D_{2 d}$ symmetry or $S_{4}$ symmetry results in the $S_{4}$ symmetry. ${ }^{11}$

For the cubic $\mathrm{KY}_{3} \mathrm{~F}_{10} / \mathrm{Eu}^{3+}$ crystal, no orientation work is needed. One has to keep in mind, however, that in the

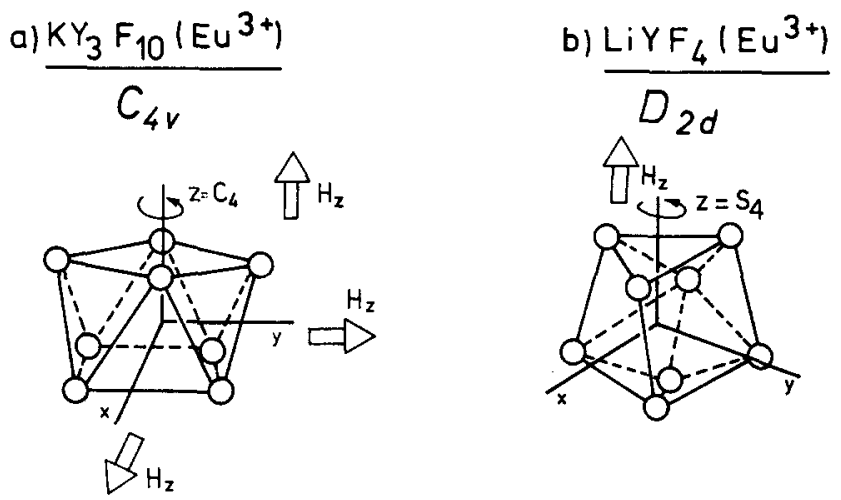

FIG. 2. Site symmetries: $2_{a}: \mathrm{KY}_{3} \mathrm{~F}_{10} / \mathrm{Eu}^{3+}\left(C_{4 v}\right), 2_{b}: \operatorname{LiYF}_{4} / \mathrm{Eu}^{3+}\left(D_{2 d}\right)$. 
crystal structure the $C_{4 v}$ sites are oriented with their $C_{4}$ axes in three directions perpendicular to each other, that is for $1 / 3$ with their tetragonal $C_{4}$ axis parallel to the magnetic field and for $2 / 3$ perpendicular to the magnetic field.

The resulting spectrum is thus expected to be a superposition of these three different sites among which only the first type fulfills the conditions required for testing the theoretical predictions of the first section of this paper. The two other sites justify the additional comments of the Sec. IV C in this paper.

\section{Recording of the MCD spectra}

The MCD spectra were recorded with a McPherson spectrophotometer (1200 lines per $\mathrm{mm}$ grating) equipped with a Morvue modulator and a 7 T supermagnet.

The MCD spectra are shown in Fig. 3 and exhibit a
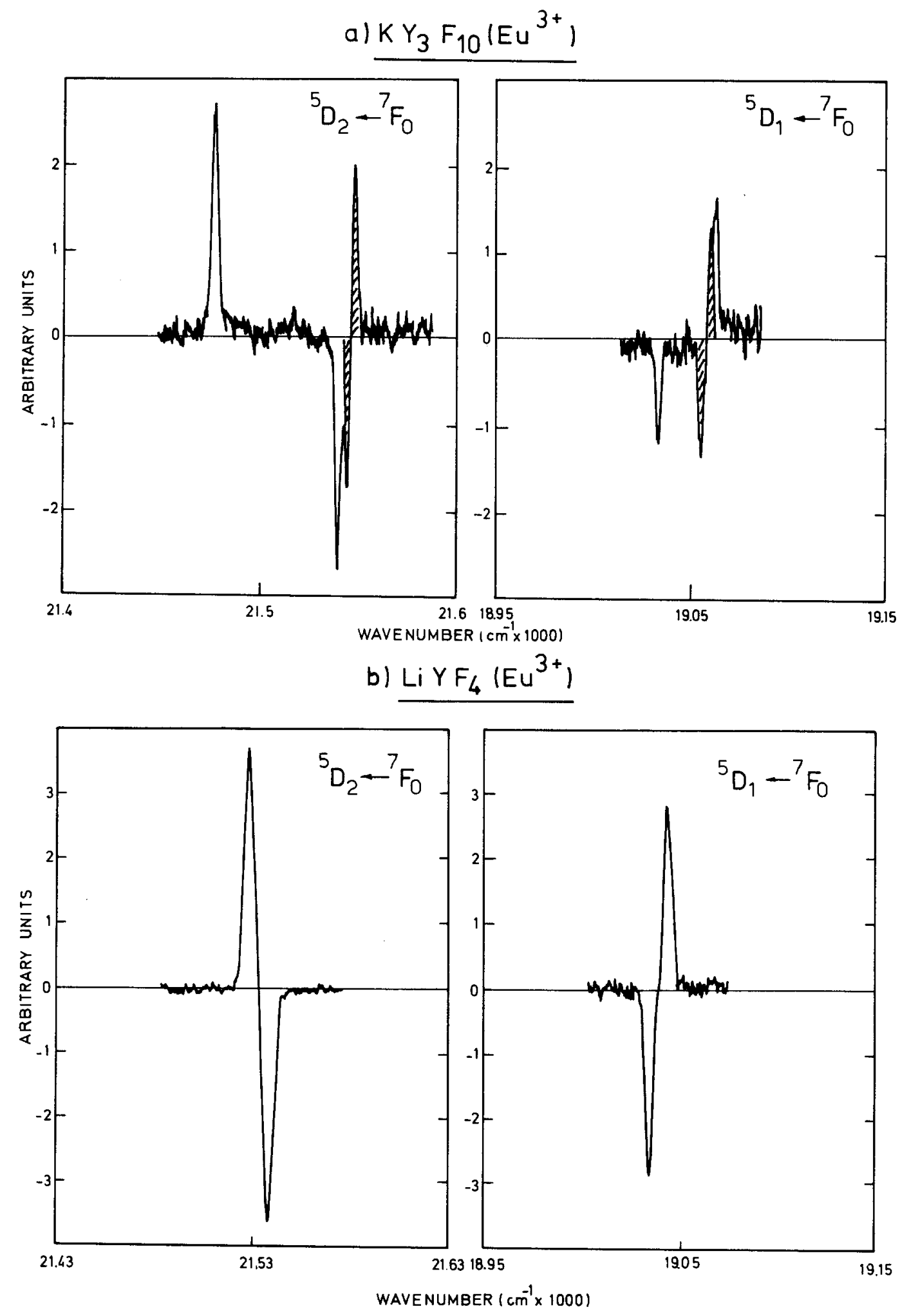

FIG. 3. MCD spectra $(293 \mathrm{~K}, 7 \mathrm{~T}): 3_{a}: \mathrm{KY}_{3} \mathrm{~F}_{10} / \mathrm{Eu}^{3+}\left(C_{4 v}\right) 3_{b}: \operatorname{LiYF}_{4} / \mathrm{Eu}^{3+}\left(D_{2 d}\right)$. 
positive $\mathscr{A}_{1}$ term for the ${ }^{5} D_{1} \leftarrow{ }^{7} F_{0}$ transition in both the $\mathrm{LiYF}_{4} / \mathrm{Eu}^{3+}$ and $\mathrm{KY}_{3} \mathrm{~F}_{10} / \mathrm{Eu}^{3+}$. The ${ }^{5} D_{2} \leftarrow^{7} F_{0}$ transition exhibits a positive $\mathscr{A}_{1}$ signal in $C_{4 v}$ while it is negative in $D_{2 d}$. Moreover, in $\mathrm{KY}_{3} \mathrm{~F}_{10} / \mathrm{Eu}^{3+} \mathscr{B}_{0}$ terms are found which show a sign inversion between ${ }^{5} D_{1} \leftarrow^{7} F_{0}$ and ${ }^{5} D_{2} \leftarrow^{7} F_{0}$ transitions. The $\mathscr{B}_{0}$ term at highest energy is positive for ${ }^{5} D_{1} \leftarrow^{7} F_{0}$ while negative for ${ }^{5} D_{2} \leftarrow{ }^{7} F_{0}$.

\section{DISCUSSION OF THE SPECTRA}

\section{A. Preliminary remarks}

The ${ }^{5} D_{1} \leftarrow{ }^{7} F_{0}$ and ${ }^{5} D_{2} \leftarrow^{7} F_{0}$ transitions are forbidden within a Russell-Saunders coupling scheme. However, due to the relaxation on the selection rules by intermediate coupling, these transitions do occur. This means that the ${ }^{5} D_{2}$ and ${ }^{5} D_{1}$ states are in fact linear combinations of states with the same $J$ value (e.g., ${ }^{5} D_{1},{ }^{7} F_{1}$, etc.... and ${ }^{5} D_{2},{ }^{7} F_{2}$, etc....).

We will first consider the $\mathscr{A}_{1}$ terms corresponding in both spectra of $\mathrm{KY}_{3} \mathrm{~F}_{10} / \mathrm{Eu}^{3+}$ and $\mathrm{LiYF}_{4} / \mathrm{Eu}^{3+}$ to the ${ }^{5} D_{1} \leftarrow^{7} F_{0}$ and ${ }^{5} D_{2} \leftarrow^{7} F_{0}$ transitions. A special section will deal with the $\mathscr{B}_{0}$ terms appearing in the spectra of the $\mathrm{KY}_{3} \mathrm{~F}_{10} / \mathrm{Eu}^{3+}$ crystais due to the fact that $2 / 3$ of the sites have their tetragonal axis perpendicular to the magnetic field.

\section{B. $\mathscr{A}_{1}$ terms}

\section{The ${ }^{5} D_{1} \leftarrow^{7} F_{o}$ transition}

Through intermediate coupling the ${ }^{5} D_{1}$ state contains to some extent ${ }^{7} F_{1}$ contribution so that for ${ }^{7} F_{1} \leftarrow^{7} F_{0}$ all the selection rules are obeyed in order to satisfy the conditions necessary for a magnetic dipole transition ${ }^{12}$ :

$$
\begin{aligned}
& \Delta l=0, \quad \Delta S=0, \Delta L=0, \\
& \Delta J=0, \pm 1(\text { not } 0 \leftrightarrow 0), \\
& \Delta M=0(\sigma \text { polarization }), \\
& \Delta \mathrm{M}= \pm 1(\pi \text { polarization }) .
\end{aligned}
$$

In Fig. 4 the crystal field splitting is given for the $C_{4 v}$ as well as the $D_{2 d}$ symmetry. In both symmetries $\pi$-polarized transitions occur from the $A_{1}$ ground state to the twofold degenerate excited $E$ state.

In the presence of a magnetic field the crystal field levels are split into an upper $\left|M^{\prime}\right\rangle=|+1\rangle$ component and a lower $|-1\rangle$ component while the ground state corresponds to $\langle\boldsymbol{M}|=\langle 0|$.

Following the selection rule $M^{\prime}-M=-\rho$, absorption of left circularly polarized light occurs to the $\left|M^{\prime}\right\rangle=|+1\rangle$ components while right circularly polarized light is absorbed to the $|-1\rangle$ component. The result is a positive $\mathscr{A}_{1}$ term in both symmetries $C_{4 v}$ and $D_{2 d}$, accordingly to the expressions of the $\mathscr{A}_{1}$ term in both symmetries:

$$
\begin{aligned}
\mathscr{A}_{1}= & \left\langle+1\left|L_{z}+2 S_{z}\right|+1\right\rangle\left|\left\langle 0\left|\mu_{-1}\right|+1\right\rangle\right|^{2} \\
& -\left\langle-1\left|L_{z}+2 S_{z}\right|-1\right\rangle \\
& \times\left|\left\langle 0\left|\mu_{+1}\right|-1\right\rangle\right|^{2} \\
= & +2\left\langle+1\left|L_{z}+2 S_{z}\right|+1\right\rangle\left|\left\langle 0\left|\mu_{-1}\right|+1\right\rangle\right|^{2} \\
= & +2 g\left|\left\langle 0\left|\mu_{-1}\right|+1\right\rangle\right|^{2} .
\end{aligned}
$$

\section{The ${ }^{5} D_{2} \leftarrow^{7} F_{o}$ transition}

In the case of the $C_{4 v}$ site, only the site with tetragonal axis along the propagation direction of the incident light and thus the magnetic field has to be considered (in this section).

Due to the mixing of ${ }^{5} F_{2}$ and ${ }^{5} D_{2}$ states the transition ${ }^{5} D_{2} \leftarrow{ }^{7} F_{0}$ is induced electric dipole allowed; the relevant selection rules being

$$
\Delta l= \pm 1, \quad \Delta S=0, \quad|\Delta L| \leqslant 6,
$$

$|\Delta J| \leqslant 6$, where if $J$ or $J^{\prime}=0$ then $|\Delta J|=2,4,6$.

From the fact that $J=0$ and from the triangular conditions in the $3 j$ symbol of formula (11),

$0 \leqslant \lambda \leqslant 2$ so that $\lambda=2$.

$$
\text { a. } K Y_{3} F_{10}\left(E u^{3+}\right)
$$
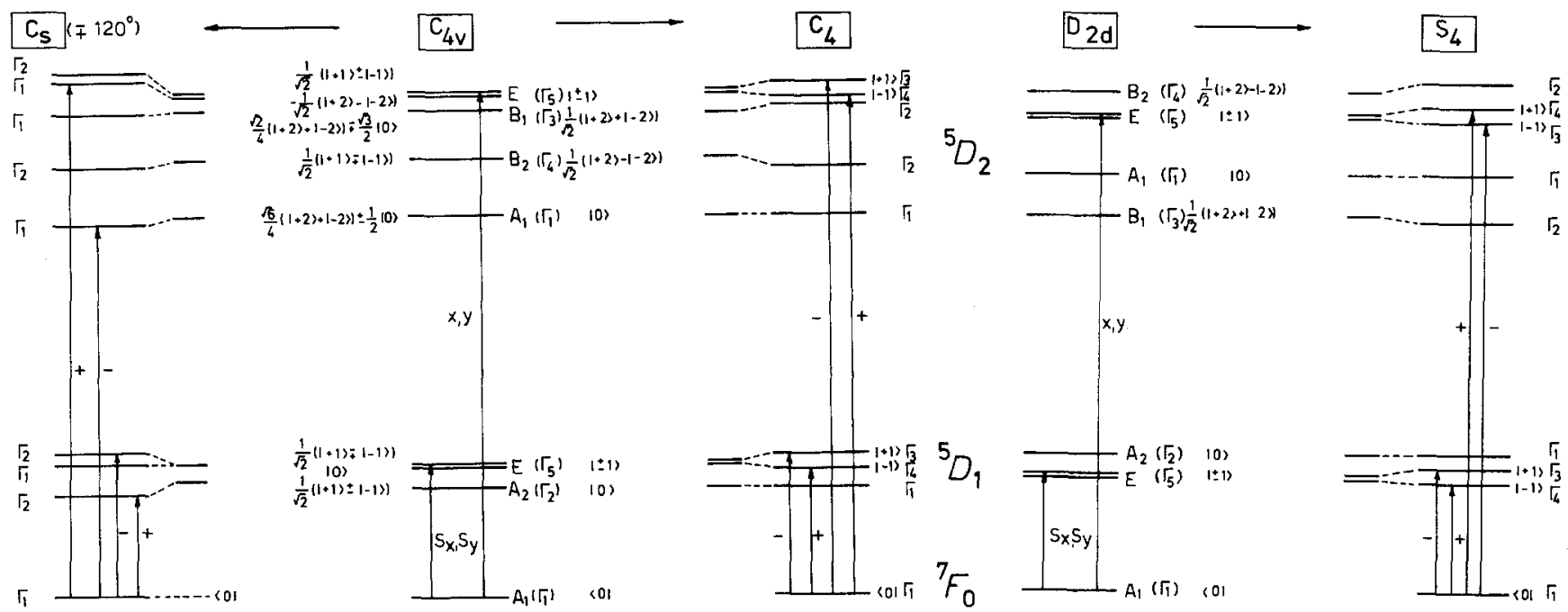

FIG. 4. Correlation scheme between crystal field and Zeeman components for the ${ }^{7} F_{0},{ }^{5} D_{1}$, and ${ }^{5} D_{2}$ levels in $D_{2 d}$ and $C_{4 v}$ site symmetry. The symbols - and + correspond to absorption of left and right circularly polarized light. $a: K_{3} \mathbf{F}_{10} / \mathrm{Eu}^{3+}, \mathrm{b}: \mathrm{LiYF}_{4} / \mathrm{Eu}^{3+}$. 
3.

The selection rules on $t$ with respect to $\lambda=2$ are $t=1$ or

The $p$ values for $t=1$ and 3 are found in the odd crystal field potential terms (see Prather ${ }^{13}$ )

$C_{4 v} A_{t p}\left(\right.$ odd): $A_{10}(C) A_{30}(C) p=0$,

$D_{2 d} A_{t p}$ (odd): $A_{32}(\mathrm{~S}) p=2$,

where $C$ and $S$ designate the tesseral harmonics (C:cos and S:sin).

In both symmetries the $E \leftarrow A_{1}$ transitions are to be considered in the light of the selection rules of Eq. (12), whereas $q=-1$, (left circularly polarized light); one obtains the following:

for $C_{4 v}, M^{\prime}=-(0-1)=+1$

leading to a positive $\mathscr{A}_{1}$ term,

for $D_{2 d} M^{\prime}=-(2-1)=-1$

leading to a negative $\mathscr{A}_{1}$ term .

The corresponding expressions of the $\mathscr{A}_{1}$ terms are, respectively, for $C_{4 v}$ symmetry:

$$
\begin{aligned}
\mathscr{A}_{1}= & \left\langle+1\left|L_{z}+2 S_{z}\right|+1\right\rangle\left|\left\langle 0\left|m_{-1}\right|+1\right\rangle\right|^{2} \\
& -\left\langle-1\left|L_{z}+2 S_{z}\right|-1\right\rangle\left|\left\langle 0\left|m_{+1}\right|-1\right\rangle\right|^{2} \\
= & +2\left\langle+1\left|L_{z}+2 S_{z}\right|+1\right\rangle\left|\left\langle 0\left|m_{-1}\right|+1\right\rangle\right|^{2} \\
= & +2 g\left|\left\langle 0\left|m_{-1}\right|+1\right\rangle\right|^{2} .
\end{aligned}
$$

For $D_{2 d}$ symmetry:

$$
\begin{aligned}
\mathscr{A}_{1}= & \left\langle-1\left|L_{z}+2 S_{z}\right|-1\right\rangle\left|\left\langle 0\left|m_{-1}\right|-1\right\rangle\right|^{2} \\
& -\left\langle+1\left|L_{z}+2 S_{z}\right|+1\right\rangle\left|\left\langle 0\left|m_{+1}\right|+1\right\rangle\right|^{2} \\
= & -2\left\langle+1\left|L_{z}+2 S_{z}\right|+1\right\rangle\left|\left\langle 0\left|m_{+1}\right|+1\right\rangle\right|^{2} \\
= & -2 g\left|\left\langle 0\left|m_{+1}\right|+1\right\rangle\right|^{2} .
\end{aligned}
$$

\section{C. $\mathscr{B}_{0}$ terms in the $\mathrm{KY}_{3} \mathrm{~F}_{10} \mathrm{MCD}$}

For the two sites having their tetragonal axis perpendicular to the direction of the magnetic field, the crystal field potential ( $\left.V=\sum_{t, p} A_{t p} D_{p}^{(t)}\right)$ has to be expressed in two other coordinate systems, obtained by cyclic permutations $x \rightarrow y \rightarrow z \rightarrow x$ (rotation of $-120^{\circ}$ ) and $x \rightarrow z \rightarrow y \rightarrow x$ (rotation of $\left.+120^{\circ}\right) .^{13}$

Once an expansion has been obtained in a given coordinate system, that in any other coordinate system may in principle be obtained from the fact that each of the terms in the new system will be expressible as a sum of terms of the same $t$ value but of different $p$ values.

The relevant transformations of the tesseral harmonics are for a rotation of $-120^{\circ}: C_{0}^{(1)} \rightarrow S_{1}^{(1)}$ and

$$
C_{0}^{(3)} \rightarrow-(\sqrt{ } 6 / 4) S_{1}^{(3)}-(\sqrt{10} / 4) S_{3}^{(3)},
$$

for a rotation of $+120^{\circ}: C_{0}^{(1)} \rightarrow C_{1}^{(1)}$ and

$$
C_{0}^{(3)} \rightarrow-(\sqrt{ } 6 / 4) C_{1}^{(3)}+(\sqrt{10} / 4) C_{3}^{(3)}
$$

The crystal field functions (see Fig. 4) are now written as linear combinations of the different $M^{\prime}$ components which means that in this case $M^{\prime}$ are no longer good quantum numbers.

Let us consider the ${ }^{5} D_{1} \leftarrow{ }^{7} F_{0}$ and ${ }^{5} D_{2} \leftarrow{ }^{7} F_{0}$ transitions.

\section{The ${ }^{5} D_{1} \rightarrow{ }^{7} F_{0}$ transition}

The application of the selection rules on transitions between the crystal field levels in Fig. 4 makes clear that magnetic dipole transitions following $S_{x}$ and $S_{y}$ occur to two levels in which $\mid+1$ ) and $\mid-1$ ) are combined (labeled $A$ " or $\Gamma_{2}$ ). One of these levels is accidently degenerate with a $|0\rangle$ level $\left(A^{\prime}\right.$ or $\left.\Gamma_{1}\right)$.

The Zeeman operator $\left(L_{z}+2 S_{z}\right)$ transforming like $S_{z}$ mixes the two $\Gamma_{2}$ states. For a rotation of $-120^{\circ}$, let us first consider the mixing of the lower state [1/ $\sqrt{ } 2)(|+1\rangle+|-1\rangle)]$ into the upper state $[(1 /$ $\checkmark 2)(|+1\rangle-|-1\rangle)]$. The energies are, respectively, $W_{K}$ for the lower and $W_{J}$ for the upper state, so that $\Delta W=W_{K}-W_{J}$ is negative.

The expression for the $\mathscr{B}_{0}$ term is

$$
\begin{aligned}
\mathscr{B}_{0}= & 2 \operatorname{Re}\left\{\frac{(1 / \sqrt{2})\left(\left\langle+1|-\langle-1|)\left|L_{z}+2 S_{z}\right|(1 / \sqrt{ } 2)(|+1\rangle+|-1\rangle)\right.\right.}{\Delta W}\right. \\
& \times\left(\left\langle0 | \mu _ { - 1 } | ( 1 / \sqrt { } 2 ) ( | + 1 \rangle - | - 1 \rangle ) ( 1 / \sqrt { } 2 ) \left(\left\langle+1|+\langle-1|)\left|\mu_{+1}\right| 0\right\rangle\right.\right.\right. \\
& -\left\langle 0\left|\mu_{+1}\right|(1 / \sqrt{ } 2)(|+1\rangle-|-1\rangle)(1 / \sqrt{ } 2)\left(\left\langle+1|+\langle-1|)\left|\mu_{-1}\right| 0\right\rangle\right\}\right. \\
= & -\frac{2 g}{\Delta W}\left|\left\langle 0\left|\mu_{-1}\right|+1\right\rangle\right|^{2} .
\end{aligned}
$$

Because $\Delta W$ is negative, the MCD signal at higher energies consists of a positive $\mathscr{B}_{0}$ term.

An analogous derivation leads to the conclusion that at lower energies the MCD signal yields a negative $\mathscr{B}_{0}$ term as $\Delta W$ is positive.

One can conclude therefore that the MCD spectrum consists of a negative $\mathscr{B}_{0}$ term to the low energy side of the unperturbed zero field transition and a positive $\mathscr{B}_{0}$ term to the high energy side; that is the composite MCD signal looks like the $\mathscr{A}_{1}$ term.

Exactly the same result is obtained when the order of the upper and lower states are reversed as it is the case for the $+120^{\circ}$ axis rotation. 


\section{The ${ }^{5} D_{2} \leftarrow{ }^{7} F_{0}$ transition}

In Fig. 4 the wave functions and energy shifts are given for the ${ }^{5} D_{2} \leftarrow^{7} F_{0}$ transition in the same way as for the ${ }^{5} D_{1} \leftarrow{ }^{7} F_{0}$ transition.

The emphasis lies now, however, on the fact that absorption [following the selection rules for the induced electric dipole mechanism $(x, y)]$ occurs to components that are linear combinations of the $|+2\rangle,|0\rangle$, and $|-2\rangle\left|M^{\prime}\right\rangle$ values. These correspond to the $A^{\prime}\left(\Gamma_{1}\right)$ in $C_{s}$, the symmetry obtained by applying the magnetic field (see Fig. 4).

By application of the Zeeman operator the same considerations hold true as for the ${ }^{5} D_{1} \leftarrow \leftarrow^{7} F_{0}$ transition, i.e., mixing of the $\Gamma_{1}$ states under $\left|\mathbf{L}_{z}+2 \mathrm{~S}_{z}\right|$, resulting in three $\mathscr{B}_{0}$ terms. For the $-120^{\circ}$ rotation these terms are as follows:

(1) to the upper level, characterized by $-(1 / \sqrt{2})(|+2\rangle-|-2\rangle)$ and $W_{J}$ corresponds:

$$
\begin{aligned}
\mathscr{B}_{0}(1)= & 2\left(\frac{-(1 / \sqrt{2})\left(\left\langle+2|-\langle-2|)\left|L_{z}+2 S_{z}\right|\{(\sqrt{2} / 4)|+2\rangle-(\sqrt{3} / 2)|0\rangle+(\sqrt{2} / 4)|-2\rangle\}\right.\right.}{W_{K_{1}}-W_{J}}\right. \\
& \times\left\{\left\langle0\left|m_{-1}\right|-(1 / \sqrt{2})(|+2\rangle-|-2\rangle)[(\sqrt{2} / 4)\langle+2|-(\sqrt{3} / 2)\langle 0|\right.\right. \\
& \left.+(\sqrt{2} / 4)\langle-2|]\left|m_{+1}\right| 0\right\rangle-\left\langle 0\left|m_{+1}\right|-(1 / \sqrt{2})(|+2\rangle-|-2\rangle)\right. \\
& \times\left[(\sqrt{2} / 4)\langle+2|-(\sqrt{3} / 2)\left\langle 0|+(\sqrt{2} / 4)\langle-2|]\left|m_{-1}\right| 0\right\rangle\right\} \\
& +\frac{-(1 / \sqrt{2})\left(\left\langle+2|-\langle-2|)\left|L_{z}+2 S_{z}\right|\left[(\sqrt{6} / 4)|+2\rangle+\frac{1}{2}|0\rangle+(\sqrt{6} / 4)|-2\rangle\right]\right.\right.}{W_{K_{2}}-W_{J}} \\
& \times\left\{\left\langle0\left|m_{-1}\right|-(1 / \sqrt{2})(|+2\rangle-|-2\rangle)\left[(\sqrt{6} / 4)\langle+2|+\frac{1}{2}\langle 0|\right.\right.\right. \\
& \left.+(\sqrt{6} / 4)\langle-2|]\left|m_{+1}\right| 0\right\rangle-\left\langle 0\left|m_{+1}\right|-(1 / \sqrt{2})(|+2\rangle-|-2\rangle)\right. \\
& \left.\times\left[(\sqrt{6} / 4)\langle+2|+\frac{1}{2}\left\langle 0|+(\sqrt{6} / 4)\langle-2|]\left|m_{-1}\right| 0\right\rangle\right\}\right),
\end{aligned}
$$

where $\mathrm{W}_{\mathrm{J}}, W_{K_{1}}$, and $W_{K_{2}}$, are, respectively, the energies of the upper, intermediate, and lower level:

$W_{K_{1}}-W_{J}<0$ and $W_{K_{2}}-W_{J}<0$.

This results in

$$
\begin{aligned}
\mathscr{B}_{0}(1)= & 2\left(( \frac { - g } { W _ { K _ { 1 } } - W _ { J } } ) \left\{\frac{1}{2}\left(\left|\left\langle 0\left|m_{-1}\right|+2\right\rangle\right|^{2}-\left|\left\langle 0\left|m_{-1}\right|-2\right\rangle\right|^{2}\right)\right.\right. \\
& \left.+(\sqrt{6} / 2)\left\langle 0\left|m_{-1}\right| 0\right\rangle\left(\left\langle 0\left|m_{-1}\right|+2\right\rangle-\left\langle 0\left|m_{-1}\right|-2\right\rangle\right)\right\} \\
& +\left(-\frac{\sqrt{3} g}{W_{K_{2}}-W_{J}}\right)\left\{(\sqrt{3} / 2)\left(\left|\left\langle 0\left|m_{-1}\right|+2\right\rangle\right|^{2}-\left|\left\langle 0\left|m_{-1}\right|-2\right\rangle\right|^{2}\right)\right. \\
& \left.\left.-(1 / \sqrt{2})\left\langle 0\left|m_{-1}\right| 0\right\rangle\left(\left\langle 0\left|m_{-1}\right|+2\right\rangle-\left\langle 0\left|m_{-1}\right|-2\right\rangle\right)\right\}\right),
\end{aligned}
$$

(2) to the intermediate level, characterized by $(\sqrt{2} / 4)|+2\rangle-(\sqrt{3} / 2)|0\rangle+(\sqrt{2} / 4)|-2\rangle$ and $W_{K_{1}}$ corresponds:

$$
\begin{aligned}
\mathscr{B}_{0}(2)= & 2\left(\frac{\left[(\sqrt{2} / 4)\langle+2|-(\sqrt{3} / 2)\left\langle 0|+(\sqrt{2} / 4)\langle-2|]\left|L_{z}+2 S_{z}\right|-(1 / \sqrt{2})(|+2\rangle-|-2\rangle)\right.\right.}{W_{J}-W_{K_{1}}}\right. \\
& \times\left\{\left\langle0\left|m_{-1}\right|[(\sqrt{2} / 4)|+2\rangle-(\sqrt{3} / 2)|0\rangle+(\sqrt{2} / 4)|-2\rangle]\right.\right. \\
& \times\left(-(1 / \sqrt{2})\left(\left\langle+2|-\langle-2|)\left|m_{+1}\right| 0\right\rangle\right)-\left\langle 0\left|m_{+1}\right|[(\sqrt{2} / 4)|+2\rangle\right.\right. \\
& \left.-(\sqrt{3} / 2)|0\rangle+(\sqrt{2} / 4)|-2\rangle]\left(-(1 / \sqrt{2})\left(\left\langle+2|-\langle-2|)\left|m_{-1}\right| 0\right\rangle\right)\right\}\right), \\
\mathscr{B}_{0}(2)= & 2\left[\frac{-g}{W_{J}-W_{K_{1}}}\right]\left[\frac{1}{2}\left(\left|\left\langle 0\left|m_{-1}\right|+2\right\rangle\right|^{2}-\left|\left\langle 0\left|m_{-1}\right|-2\right\rangle\right|^{2}\right)+(\sqrt{6} / 2)\left\langle 0\left|m_{-1}\right| 0\right\rangle\right. \\
& \left.\times\left(\left\langle 0\left|m_{-1}\right|+2\right\rangle-\left\langle 0\left|m_{-1}\right|-2\right\rangle\right)\right],
\end{aligned}
$$

where $W_{J}-W_{K_{1}}>0$;

(3) to the lower level, characterized by $(\sqrt{6} / 4)|+2\rangle+\frac{1}{2}|0\rangle+(\sqrt{6} / 4)|-2\rangle$ 


$$
\begin{aligned}
\mathscr{B}_{0}(3)= & 2\left(\frac{\left[(\sqrt{6} / 4)\langle+2|+\frac{1}{2}\left\langle 0|+(\sqrt{6} / 4)\langle-2|]\left|L_{z}+2 S_{z}\right|-(1 / \sqrt{2})(|+2\rangle-|-2\rangle)\right.\right.}{W_{J}-W_{K_{2}}}\right. \\
& \times\left(\left\langle0\left|m_{-1}\right|\left[(\sqrt{6} / 4)|+2\rangle+\frac{1}{2}|0\rangle+(\sqrt{6} / 4)|-2\rangle\right]\right.\right. \\
& \times\left(-(1 / \sqrt{2})\left(\left\langle+2|-\langle-2|)\left|m_{+1}\right| 0\right\rangle\right)-\left\langle0 | m _ { + 1 } | \left[(\sqrt{6} / 4)|+2\rangle+\frac{1}{2}|0\rangle\right.\right.\right. \\
& \left.+(\sqrt{6} / 4)|-2\rangle]\left(-(1 / \sqrt{2})\left(\left\langle+2|-\langle-2|)\left|m_{-1}\right| 0\right\rangle\right)\right\}\right), \\
\mathscr{B}_{0}(3)= & 2\left[-\frac{\sqrt{3} g}{W_{J}-W_{K_{2}}}\right]\left[\frac{\sqrt{3}}{2}\left(\left|\left\langle 0\left|m_{-1}\right|+2\right\rangle\right|^{2}-\left|\left\langle 0\left|m_{-1}\right|-2\right\rangle\right|^{2}\right)\right. \\
& \left.+(1 / \sqrt{2})\left\langle 0\left|m_{-1}\right| 0\right\rangle\left(-\left\langle 0\left|m_{-1}\right|+2\right\rangle+\left\langle 0\left|m_{-1}\right|-2\right\rangle\right)\right],
\end{aligned}
$$

where $W_{J}-W_{K_{2}}>0$.

The situation is complicated to some extent by the fact that the sign of the $\mathscr{B}_{0}$ term is the result of the interplay of different intensity matrix elements.

By looking at the odd crystal field terms, which occur in the expansion of the crystal field potential after a consideration of the coordinate system transformation:

$$
\begin{array}{lll}
A_{t p}\left(\text { odd) } \quad A_{11} C(\mathrm{~S})\right. & A_{31} C S & p=1 \\
& A_{33} C S & p=3
\end{array}
$$

and at the selection rule $M^{\prime}=-(q+p)$, it is found that to $|-2\rangle$ absorption of left circularly polarized light occurs through the $A_{33}$ parameter, while absorption of right circularly polarized light takes place through $A_{11}$ and $A_{31}$. To $|+2\rangle$, exactly the inverse holds true.

Moreover, the $A_{11}$ and $A_{31}$ are effective in the absorption of left and right circularly polarized light to the $|0\rangle$ component to the same extent. Calculations based on the intensities of fluorescence spectra by Porcher and Caro show that the $A_{33} \gg A_{31}$ and $A_{11}$, so that the matrix element $\left\langle 0\left|m_{-1}\right|-2\right\rangle$ is greater than $\left\langle 0\left|m_{-1}\right|+2\right\rangle$. The detailed calculations will be given extensively in a second paper that takes into account the wave functions in an extended, $J-J$ coupling, basis set. ${ }^{17}$

However we can summarize the result of the calculations as follows: all the electric dipole matrix elements for the $-120^{\circ}$ rotation are imaginary. Numerically the second part of the $\mathscr{B}_{0}(1)$ term [with coefficient $-\sqrt{3} g /\left(W_{K_{2}}-W_{J}\right)$ ] turns out to be predominant, as it contains a sum of two terms, whereas the difference of these same two terms appears in the first part.

Finally, as mentioned previously in this second section, the terms $\left|\left\langle 0\left|m_{-1}\right|-2\right\rangle\right|^{2}$ and $\left\langle 0\left|m_{-1}\right| 0\right\rangle\left\langle 0\left|m_{-1}\right|-2\right\rangle$ have the largest contribution. The $\mathscr{B}_{0}(1)$ term has thus the sign of $W_{K_{2}}-W_{J}<0$. As a consequence the related $\mathscr{B}_{0}(3)$ term is positive since it contains $W_{J}-W_{K_{2}}>0$.

$\mathscr{B}_{0}(2)$ is related (but of opposite sign) to the first part of $\mathscr{B}_{0}(1)$ [with coefficient $-g /\left(W_{K_{1}}-W_{J}\right)$ ]; our calculations indicate that it is one or two orders of magnitude smaller than the two other $\mathscr{B}_{0}$ terms and indeed difficult to detect experimentally.

\section{CONCLUSION}

The central objective of this paper is to show that a purely qualitative sign argumentation for induced electric dipole transition in MCD spectra can lead to definite conclusions about the site symmetry of the coordination sphere surrounding the rare earth ion.

The case illustrated here demonstrates how the $C_{4 v}$ symmetry can unambiguously be differentiated from the $D_{2 d}$ symmetry by the sign of the $\mathscr{A}_{1}$ terms associated to the ${ }^{5} D_{2} \leftarrow^{7} F_{0}$ induced electric dipole transition. We recall that the magnetic dipole transition ${ }^{5} D_{1} \leftarrow{ }^{7} F_{0}$ shows the same sign for the $\mathscr{A}_{1}$ term in both symmetries.

With respect to this the ${ }^{5} D_{1} \leftarrow^{7} F_{0}$ can serve as a "sign reference transition" and the ${ }^{5} D_{2} \leftarrow{ }^{7} F_{0}$ can be used as a "MCD probe" (in addition to the well-established NMR and fluorescence probes) for testing the coordination structure.

We wish to emphasize that such a simple differentiation between $D_{2 d}$ and $C_{4 v}$ symmetries cannot be obtained by absorption or fluorescence measurements, and even by polarization spectroscopy; indeed in both cases the spectra will show (both for $x-y$ absorption or emission) the same kind of signal due to a transition from the nondegenerate ground state to a degenerate $E$ state.

The case worked out here theoretically was chosen ad $h o c$ to test the validity of the selection rules and their consequences for the sign of the $\mathscr{A}_{1}$ terms in a rather simple way. The most difficult step was, however, to choose the single crystal systems that enabled us to confirm experimentally the predicted theory.

The presence of two other sites in the $\mathrm{KY}_{3} \mathrm{~F}_{10} / \mathrm{Eu}^{3+}$ crystal although requiring a more complicated theoretical development, allows one to draw some clear conclusions on the low symmetry of $C_{S}$.

${ }^{1}$ C. Görller-Walrand and J. Godemont, J. Chem. Phys. 66, 48 (1977).

${ }^{2}$ B. R. Judd, Phys. Rev. 127, 750 (1962).

${ }^{3}$ W. T. Carnall, P. R. Fields, and K. Rajnak, J. Chem. Phys. 49, 10, 4412 (1968).

${ }^{4}$ P. J. Stephens, Adv. Chem. Phys. 35, 197 (1976).

${ }^{5}$ S. B. Piepho and P. N. Schatz, Group Theory in Spectroscopy with Applications to Magnetic Circular Dichroism (Wiley, New York, 1983).

${ }^{6}$ G. S. Ofelt, J. Chem. Phys. 37, 511 (1962).

${ }^{7}$ M. P. Borzenkova, G. N. Kuznetsova, and A. V. Novoselova, Izv. Akad. Nauk. SSSR Neorg. Mater. 7, 242 (1971).

${ }^{8}$ I. Laursen and L. M. Holmes, J. Phys. C 7, 3765 (1964).

${ }^{9}$ R. E. Thoma, G. D. Brunton, R. A. Penneman, and T. K. Keenan, Inorg. Chem. 9, 5, 1096 (1970).

${ }^{10} \mathrm{~J}$. W. Pierce and H. Y. L. Hong, Proc. 10th Rare Earthly Res. Conf. 1973, $527(1974)$. 
${ }^{11}$ G. F. Koster, J. O. Dimmock, R. G. Wheeler, and H. Statz, Properties of the thirty-two point groups (MIT, Cambridge, Mass., 1963).

${ }^{12} \mathrm{G}$. H. Dieke, Spectra and Energy Levels of Rare Earth Ions in Crystals (Interscience, New York, 1968).

${ }^{13}$ J. L. Prather, Atomic Levels in Crystals, Natl. Bur. Stand Monogr. 19 (U.S. GPO, Washington, D.C., 1961).

${ }^{14}$ C. Görller-Walrand, M. Behets, P. Porcher, O. K. Moune-Minn, and I.
Laursen, Inorg. Chim. Acta 109, 83 (1985).

${ }^{15}$ P. Porcher and P. Caro, J. Chem. Phys. 65, 1, 89 (1976).

${ }^{16}$ P. Porcher and P. Caro, J. Chem. Phys. 68, 9, 4176 (1978).

${ }^{17}$ P. Porcher and P. Caro, J. Lumin. 21, 207 (1980).

${ }^{18} \mathrm{C}$. Göller-Walrand and L. Fluyt-Adriaens, J. Less Common Met. (submitted).

${ }^{19}$ R. D. Peacock, Struct. Bond. Berlin 22, 83 (1975). 